\title{
Intersectorialidad y reducción del estigma en salud mental: la necesidad de avanzar hacia una descolonización de la institucionalidad, desde una perspectiva intercultural
}

\section{Intersectoriality and reduction of stigma in mental health: the need to move towards a decolonization of institutions, from an intercultural perspective}

\section{Cristian Valderrama-Núñez ${ }^{\mathrm{a}}$}

(D) https://orcid.org/0000-0002-2526-8033

E-mail: cvalderramaœunab.cl

\section{Roxanna Zolezzi-Gorziglia ${ }^{b}$}

(1) https://orcid.org/0000-0001-8932-4594

E-mail: roxanna.zolezzi®unab.cl

anniversidad Andrés Bello, Facultad de Ciencias de la Rehabilitación. Escuela de Terapia Ocupacional. Concepción, Bio Bio, Chile.

bUniversidad Andrés Bello, Facultad de Ciencias de la Rehabilitación. Escuela de Terapia Ocupacional. Santiago, Región Metropolitana, Chile

\section{Correspondencia}

Cristian Valderrama-Núñez.

Autopista Concepcion, Talcahuano 7 100. Biobío, Chile. CEP 8370146.

\section{Resumen}

El objetivo de este estudio es analizar las implicancias en la subjetividad y los cambios en la vida cotidiana, de quienes participaron en calidad de asistentes a las Jornadas Anti-estigma en la ciudad de Concepción en Chile, una práctica socioeducativa, de carácter interinstitucional e interdisciplinar, cuyo propósito es generar un cambio cultural sobre la estigmatización en el campo de la salud mental. Se desarrolla una sistematización de esta experiencia, enfatizando en la historia y las metodologías aplicadas en ella. La información para el análisis se produce a través de un grupo de discusión con 14 participantes. Los resultados muestran que existe un impacto en la subjetividad de quienes asisten dado que se propicia el contacto con los sujetos estigmatizados y con sus experiencias de vida en torno a éste. Sin embargo, se presenta como un desafío la transformación de prácticas en los espacios cotidianos, aspectos que requieren de esfuerzos intersectoriales y una institucionalidad que genere las bases y desarrollo de políticas públicas adecuadas para ello. En este sentido resulta imperativo incluir perspectivas y prácticas decoloniales e interculturales en el campo de la salud mental.

Palabras-clave: Intersectorialidad; Estigma social; Salud Mental; Interculturalidad. 
The objective of this study is to analyze the implications on subjectivity and changes in daily life, of those who participated as attendees at the Anti-stigma Conference in the city of Concepción in Chile, a socio-educational practice, of an interinstitutional and interdisciplinary nature, whose purpose is to generate a cultural change on stigmatization in the field of mental health. A systematization of this experience is developed, emphasizing the history and the methodologies applied in it. The information for the analysis is produced through a discussion group with 14 participants. The results show that there is an impact on the subjectivity of those who attend, since contact with stigmatized subjects and with their life experiences around them is encouraged. However, the transformation of practices in everyday spaces is presented as a challenge, aspects that require intersectoral efforts and an institutional framework that generates the bases and development of adequate public policies for this. In this sense, it is imperative to include decolonial and intercultural perspectives and practices in the field of mental health.

Keywords: Intersectorial colaboration; Social stigma; Mental Health; cultural competency.
En el ámbito de la salud mental, el estigma es ampliamente reconocido y así lo avalan numerosas investigaciones sobre la temática. Diversos autores han contribuido al entendimiento del estigma social, entre ellos, Michaels, López, Rüsch (2012) señalan que el estigma se caracteriza por ser un proceso sociocultural, en el cual cierto grupo de personas son etiquetados/as por otros/as, ya que presentarían características y atributos que no son socialmente aceptables o "normales". Desde esta perspectiva, el estigma es la expresión de una sociedad estructurada en base a relaciones de poder en el cual los y las llamadas "locos/as" son inferiorizados/as y subalternizados/as.

Simultáneamente, a través de lo que se conoce como auto estigma o estigma internalizado, es posible identificar los efectos negativos del estigma sobre la subjetividad de quienes lo padecen, perjudicando la autoestima y la autopercepción (Nascimento; Leão, 2019), aumentando las posibilidades de riesgo suicida por la desesperanza y devaluación que produce en las personas que lo sufren (Touriño; Acosta; Giráldez et al., 2018), internalizando el rol de enfermo en desmedro del rol de ciudadano o sujeto de derechos (Badallo; Pérez; Bertina, 2018). Por último, produce discriminación, exclusión social y dificultades para el ejercicio de la ciudadanía, afectando de manera sustancial a las personas con algún diagnóstico psiquiátrico (Aparecido; Silva, 2020).

Dada las implicancias del estigma en personas con diagnóstico psiquiátrico, es fundamental generar acciones que avancen en la reducción del estigma y sus consecuencias. Autores como Mehta, Clement, Marcus et al. (2015) y Agrest, Mascayano; Ardila-Goméz (2015) plantean la importancia de revisar y avanzar en el desarrollo de estrategias locales y de políticas públicas que puedan dar sustento a una perspectiva transversal sobre la problemática del estigma.

Según Campos, Oviedo y Herazo (2014) y Bang (2014) para el desarrollo de estrategias de reducción del estigma, es necesario un proceso estructurado de sensibilización y educación de los distintos colectivos y comunidades, con el fin de promover el conocimiento y favorecer el trabajo intersectorial. 
Además refieren que las intervenciones deben ser consistentes en el tiempo, usar diversas metodologías e incorporar los distintos sectores de la sociedad (intersectorial) incluyendo el fortalecimiento de redes comunitarias y territoriales.

En este sentido, este estudio ofrece una experiencia de reducción del estigma, que recoge las sugerencias recién expuestas, desde una perspectiva interinstitucional, interdisciplinaria y territorial. Nos referimos a las Jornadas Antiestigma, actividad que se desarrolla desde el año 2011 en la ciudad de Concepción, en la región del Bio Bio, Chile, la que podría ser una experiencia que permita avanzar hacia la descolonización de las prácticas y saberes que han desarrollado históricamente las instituciones, organizaciones sociales y la sociedad en su conjunto, en torno a la reducción del estigma de personas con diagnóstico psiquiátrico.

\section{Sobre las Jornadas Antiestigma}

Las Jornadas Antiestigma se caracterizan por desarrollar metodologías participativas, dialógicas, colaborativas y asociativas entre todos los actores sociales involucrados en su ejecución, es decir, tanto a nivel de su organización como de su propuesta temática y de las metodologías con las cuales se desarrolla (Fernández; Vinencio; Vallejos et al., 2016). Lo anterior ha sido posible dado que los principios que circulan entre los organizadores son la solidaridad, la equidad y la confianza, los cuales consideramos valores importantes para el trabajo en equipo.

Otros atributos que poseen estas Jornadas son su carácter interdisciplinar e interinstitucional, ya que participan en la organización de éstas representantes de distintas instituciones y disciplinas del ámbito de la educación como son la Universidad de Concepción (depto. de psicología) y Universidad Andrés Bello (carrera de Terapia Ocupacional), de la salud, como la Secretaría Regional Ministerial de salud del Bio Bio (SEREMI) y el Servicio de Salud Talcahuano, y del campo de la protección social, como Fundación Rostros Nuevos del Hogar de Cristo. Al mismo tiempo, las organizaciones sociales, como la agrupación ex usuarios de salud mental (AESAM) y la Corporación Voces. Dado que el estigma es un problema social complejo, requiere esfuerzos coordinados y una diversidad de saberes y prácticas para su reducción. En tal sentido, las Jornadas antiestigma aúnan voluntades para avanzar en dicho propósito; el objetivo de éstas es generar un espacio de encuentro que permita el intercambio de opiniones y experiencias, para el desarrollo de un cambio cultural sobre la estigmatización y la promoción de la salud mental y los derechos humanos.

A continuación, se presentan algunas características correspondientes a la organización de las Jornadas, para luego dar cuenta de las metodologías que se desarrollan y los hitos más relevantes en los años 2016 - 2018.

El comité intersectorial de las Jornadas es el grupo de personas que, representando a las instituciones y organizaciones mencionadas anteriormente, diseñan, planifican, ejecutan y evalúan las Jornadas. A través de reuniones sistemáticas, se juntan para monitorear los avances y el cumplimiento de las responsabilidades comprometidas por sus integrantes. Todos/as ellos/as ponen a disposición sus saberes, experiencias y redes de contacto para la elaboración del programa a desarrollar.

Este proyecto contempla 3 actividades que se desarrollan durante el año; primero, un concurso de buenas prácticas inclusivas, que permite visibilizar experiencias exitosas en la reducción del estigma. La segunda actividad que se ejecuta son talleres de capacitación, un espacio que permite la reflexión y profundización de temáticas asociadas al tema central que se abordará durante ese año, incluye la participación en calidad de relatores de expertos por experiencia, académicos y profesionales de la salud mental; por último, la tercera actividad corresponde a las Jornadas propiamente dichas, en las cuáles se presentan desde distintas perspectivas y experiencias el tema central respectivo. Todas las actividades mencionadas están dirigidas a los equipos de salud y de salud mental, académicos, estudiantes, dirigentes de organizaciones sociales y comunitarias, entre otros.

Los/as expositoras/es y expertas/os por experiencias invitadas/os participan de manera voluntaria, y la organización cubre los gastos de pasajes aéreos, estadía y alimentación según corresponda.

Las Jornadas Antiestigma metodológicamente despliegan una variedad de estrategias de enseñanza 
con la pretensión de surtir efecto en los asistentes a ellas, entre las que se destacan, se pueden mencionar los talleres como un espacio para el análisis de casos, para desarrollar aprendizajes activos y significativos, a través de la resolución de problemas de forma cooperativa.

Se desarrollan exposiciones para facilitar procesos de reflexión en las materias de interés. Para fomentar el aprendizaje de las vivencias en primera persona, estando en una relación simétrica, se realizan conversatorios con invitados definidos como “expertos/as por experiencia”. Esta denominación se atribuye a las personas con diagnósticos psiquiátricos y/o sus familiares, quienes son considerados expertos de la problemática de salud y de los alcances familiares y sociales de ésta, ya que son los que viven permanentemente con ella, y sobretodo constituyen su identidad, es decir configura su subjetividad reconociendo sobre todo su condición de un otro/a legítimo/a y auténtico/a (Salas, 2017; Castillo, 2018; Cea-Madrid; Parada, 2018). Para llevar a cabo estos conversatorios, se diseña un guion de entrevista que se presenta con anterioridad a los participantes para que puedan hacer observaciones y participen en la construcción de los temas a tratar en el espacio.

En el caso de las exposicionesy de los conversatorios, se destina tiempo para que los/as asistentes puedan plantear preguntas o hacer comentarios sobre los temas y experiencias presentadas, a este espacio se le denomina plenarios. Este espacio es relevante en tanto, permite el intercambio de saberes, experiencias e historias de vida no solo de los expositores sino que también de los asistentes, quienes las comparten voluntariamente, enriqueciendo los aprendizajes de todos/as de manera colectiva.

Otras estrategias incorporadas durante estos últimos años son el teatro que permite recrear situaciones cotidianas de estigmatización, para, desde ahí, humanizar los efectos de la discriminación apelando a la comprensión afectiva que provoca este método. Este método se implementa de dos formas: con la presentación de obras de teatro sobre las vivencias de personas que poseen un diagnóstico, y por otro lado dramatizando una historia vinculada a la temática, durante el desarrollo de las Jornadas, en las cuales participan estudiantes universitarios, los organizadores y expertos por experiencias. Se han construido videos y otros materiales para difundir y promover la ciudadanía.

Por último, se ha incorporado la autoetnografía (Guerrero, 2014), para ofrecer testimonios en primera persona de la vivencia de estigma y las implicancias en la vida cotidiana destacando los aspectos emocionales y subjetivos de dicha experiencia, para comprender desde esta perspectiva la magnitud social del fenómeno del estigma.

Todas las estrategias y metodologías utilizadas en las Jornadas pueden ser consideradas sugerencias concretas para otras instituciones u organizaciones sociales que tienen entre sus objetivos generar prácticas para la superación del estigma.

Para los propósitos de este texto, se torna relevante a partir de lo que señalan Fernández, Vinencio, Vallejos et al. (2016) que las intervenciones de reducción del estigma requieren considerar y evaluar los cambios a nivel subjetivo que producen estas acciones y cómo los sujetos participantes implementan cambios en sus cotidianidades y en las comunidades. A lo anterior, se suma lo planteado por Salas (2017), quien señala que los programas educativos para reducir el estigma, que permitan la discusión, presentación de casos y la presencia de personas afectadas resultan más efectivos en ese propósito. Considerando los fundamentos recién planteados, este estudio se propone como objetivo analizar los efectos que han producido las Jornadas antiestigma en las subjetividades, y de qué manera esta experiencia permea las prácticas cotidianas de los asistentes a ellas.

Para ello, se ha definido sistematizar las tres últimas Jornadas realizadas durante los años 2016 - 2018. Esta decisión se toma dado que durante estos años se realizan cambios importantes en las metodologías con las cuales se desarrollan los temas seleccionados. Por otro lado, durante este periodo las Jornadas cuentan con recursos permanentes para su desarrollo, y por último, el comité organizador considera que luego de tres años es necesario reflexionar sobre las prácticas desarrolladas, con el propósito de mejorar la gestión de estas Jornadas. 
Tabla I - Jornadas anti estigma años 2016-2018

\begin{tabular}{|c|c|c|c|c|}
\hline Año & Tema de las Jornadas & Expositores & Subtemas & Metodologías \\
\hline 2016 & $\begin{array}{l}\text { Desestigmatización } \\
\text { de la Infancia: } \\
\text { "Rotulación de } \\
\text { la infancia como } \\
\text { una vulneración } \\
\text { de derechos". }\end{array}$ & $\begin{array}{l}\text { Instituto de derechos } \\
\text { Humanos (INDH). } \\
\text { Agrupación de ayuda a niñas y } \\
\text { niños autistas (AGANAT). } \\
\text { Depto. Psicología Universidad } \\
\text { de Concepción. } \\
\text { Instituto Chileno de } \\
\text { Terapia Familiar. } \\
\text { Fundación Tierra esperanza. } \\
\text { Organización no } \\
\text { gubernamental (ONG) } \\
\text { Poder compartido. }\end{array}$ & $\begin{array}{l}\text { Principios de Igualdad y No } \\
\text { discriminación en la Infancia } \\
\text { Obra de teatro "nosotros nos } \\
\text { hacemos cargo" } \\
\text { Sobrediagnóstico en infancia y } \\
\text { adolescencia. Los efectos, riesgos } \\
\text { y alcances de una práctica común } \\
\text { Estigma en familias vulneradas. } \\
\text { Algunas reflexiones sobre el } \\
\text { trabajo con familias y sus hijos. } \\
\text { Adolescencia, delito y estigma } \\
\text { Adultismo y adultocentrismo. } \\
\text { Metodologías y prácticas de } \\
\text { participación que favorecen } \\
\text { la inclusión de la voz } \\
\text { infanto adolescente }\end{array}$ & $\begin{array}{l}\text { Talleres } \\
\text { Exposiciones } \\
\text { Conversatorios } \\
\text { Plenarios } \\
\text { Obra de teatro } \\
\text { Grupos de discusión }\end{array}$ \\
\hline 2017 & $\begin{array}{l}\text { "Des-cubriendo el } \\
\text { estigma en la vida } \\
\text { cotidiana" }\end{array}$ & $\begin{array}{l}\text { Depto. Psicología Universidad } \\
\text { de Concepción. } \\
\text { Escuela de Terapia } \\
\text { Ocupacional Universidad } \\
\text { Andrés Bello. } \\
\text { Centro de Rehabilitación } \\
\text { Diurno "Luz de Luna". }\end{array}$ & $\begin{array}{l}\text { "No es lo mismo ser loca que } \\
\text { loco: No sé cómo llegué aquí, } \\
\text { pero sé cómo salir". } \\
\text { Auto etnografía, un testimonio } \\
\text { en primera persona sobre } \\
\text { el estigma. } \\
\text { Experiencias de recuperación I. } \\
\text { Experiencias de recuperación } 2 .\end{array}$ & $\begin{array}{l}\text { Talleres } \\
\text { Exposiciones } \\
\text { Conversatorios } \\
\text { Plenarios } \\
\text { Autoetnografía } \\
\text { Teatro } \\
\text { Video disponible en: } \\
\text { https://www.youtube. } \\
\text { com/watch?v=zyIR4-TkEko }\end{array}$ \\
\hline 2018 & $\begin{array}{l}\text { "Trabajo, estigma } \\
\text { y salud mental: del } \\
\text { camino recorrido } \\
\text { al camino por } \\
\text { recorrer" }\end{array}$ & $\begin{array}{l}\text { Fundación SOL. } \\
\text { Escuela de Terapia } \\
\text { Ocupacional Universidad de } \\
\text { Santiago de Chile. } \\
\text { Red de Inclusión Bio Bio. } \\
\text { Colectivo Locos por nuestros } \\
\text { derechos. } \\
\text { Expertos/as por experiencias } \\
\text { incluidos/as laboralmente. }\end{array}$ & $\begin{array}{l}\text { Trabajo, situación actual en Chile. } \\
\text { Trabajo, derechos humanos y } \\
\text { salud mental. } \\
\text { Avances y desafíos en la } \\
\text { inclusión al trabajo. } \\
\text { "Trabajar como loco": } \\
\text { cooperativismo y autogestión en } \\
\text { salud mental. } \\
\text { Experiencias de inclusión } \\
\text { al trabajo. }\end{array}$ & $\begin{array}{l}\text { Talleres } \\
\text { Exposiciones } \\
\text { Conversatorios } \\
\text { Plenarios } \\
\text { Autoetnografía } \\
\text { Teatro } \\
\text { Videos (narrativas } \\
\text { de experiencias de } \\
\text { inclusión laboral) }\end{array}$ \\
\hline
\end{tabular}


Desde una mirada decolonial, es posible señalar que las Jornadas Antiestigma presenta características que avanzan en esta racionalidad, entre las que se pueden mencionar una variedad significativa de metodologías participativas en el proceso de enseñanza y aprendizaje en la reducción del estigma, ser una plataforma para reconocer la voz de las personas con diagnóstico psiquiátrico y, por último, considerar a las personas estigmatizadas como ciudadanos, como sujetos de derechos.

Lo anterior convive y se configura con los saberes y prácticas de la racionalidad propias de las instituciones que la organizan, entre ellas, la lógica del proyectismo y el tecnicismo para obtener y rendir los recursos financieros y materiales que las permiten Otra expresión es que las Jornadas se ejecutan en espacios académicos en los que tienden a dominar los saberes de expertos y el saber legítimo, por sobre el saber popular o de la experiencia. De acuerdo a lo recién descrito, se requiere un ejercicio permanente de reflexión para evitar que estas condiciones limiten el ejercicio decolonial en el campo del estigma que esta actividad propone.

\section{Metodología}

La propuesta metodológica para abordar el objetivo de este estudio, corresponde a una sistematización, ya que ésta busca rescatar de manera reflexiva y crítica los aprendizajes que se producen en una experiencia vivida y, desde ahí, fomentar praxis transformadoras de quienes participan en el proceso (Expósito; González, 2017). Esta metodología resulta pertinente, en tanto, las Jornadas Antiestigma son una actividad socioeducativa que produce aprendizajes, los cuales circulan entre los involucrados en ella, que requieren ser reflexionados, compartidos y ordenados para dar significado, sentido y orientación en los pasos a seguir en el camino inconcluso en el cual se inscribe.

Esta sistematización incluyó la revisión de una serie de documentos construidos en la planificación, ejecución y evaluación de las Jornadas, por parte del comité intersectorial, específicamente los programas de las Jornadas, actas de reuniones, afiches, con los cuales se reconstruyeron los hechos relevantes de las Jornadas durante el periodo 2016-2018, presentados previamente en este texto.
Para el objetivo de esta investigación, se torna relevante la opinión de los asistentes a las Jornadas, por tal motivo se acude a un muestreo cualitativo por conveniencia, se establecieron ciertos criterios de inclusión, entre los que se encuentran: haber participado por lo menos en una de las últimas tres Jornadas realizadas (2016-2018) y representar a algunos de los sectores involucrados habitualmente en las Jornadas (educación, salud, protección social, agrupación de usuarios y familiares).

Para conformar al grupo de participantes, se revisaron las listas de asistencia a talleres y a las Jornadas. Luego de la identificación según los criterios preestablecidos, 3 integrantes del comité intersectorial hicieron contacto telefónico y/o presencial con los potenciales participantes, para explicar los objetivos de la investigación. Si éstos declaraban interés y voluntariedad en participar, se formalizó invitación a través de correo electrónico. Se convocaron a 36 personas, de las cuales 14 participaron de este estudio y se distribuyen de la siguiente manera: 2 docentes de educación (1 educación adultos, 1 de educación superior), 3 estudiantes universitarios (2 de Psicología y 1 de Terapia Ocupacional), 5 funcionarios de salud (4 de dispositivos de salud mental y 1 de atención primaria de salud), 2 representantes de agrupación de usuarios y familiares de salud mental, 2 funcionarios de protección social (oficina municipal de la discapacidad). Con ello, se encuentran presentes en el estudio todos los sectores que habitualmente participan en las Jornadas. En relación a la distribución por género: 4 de los participantes son hombres y 10 de las participantes son mujeres.

En relación a los participantes de este estudio es relevante indicar que todos son de nacionalidad chilena, pertenecen a distintos grupos etarios, pertenecen a la clase obrera trabajadora y principalmente son mujeres. Desde una perspectiva decolonial, estos atributos representan las características habituales de quienes se dedican a los cuidados, estudios e intervenciones de personas con diagnóstico psiquiátrico en el marco de la institucionalidad, por tanto sus saberes y prácticas dan cuenta de la realidad actual en torno al tema.

Para la producción de la información se utilizó un grupo de discusión, ya que esta técnica permite, a través del intercambio de opiniones y perspectivas, 
reconstruir las experiencias individuales y colectivas y, al mismo tiempo, los participantes pueden influir mutuamente entre ellos, para modificar sus actitudes, percepciones y, con ello, saberse parte de un sentido común que se construye durante el dialogo grupal (Archenti, 2018). Se elaboró y aplicó un guion temático construido por el comité organizador y validado por expertas/os, el cual se organizó con las siguientes temáticas: fortalezas y debilidades de las Jornadas, metodologías utilizadas, aprendizajes e incorporación de los temas y metodologías en la práctica cotidiana. El grupo de discusión fue liderado por 3 integrantes del comité intersectorial que no participaron de la convocatoria, para evitar influencia en los resultados del estudio.

El grupo de discusión fue grabado en audio y transcrito de manera textual. El análisis de la información se desarrolló a través de un análisis de contenido, a partir de un proceso de codificación abierta (Santander, 2011), en el cual se agruparon los discursos de los participantes, de acuerdo al sector al cual pertenecen: educación (ED), profesionales de salud (S), protección social (PS) y agrupaciones comunitarias (AGC). Posteriormente, se organiza la información considerando las siguientes temáticas: impactos personales y cambios en las prácticas, para simplificar la información. Al mismo tiempo, se establecen códigos que identifican el sector al cual pertenece el participante, dado que el discurso personal es considerado el discurso social que circula en los distintos grupos, es decir, el discurso contiene propiedades subjetivas, pero al mismo tiempo expresa la intersubjetividad en la cual es posible, por tanto denota simultáneamente la individualidad y colectividad de quien lo emite. Finalmente, para el proceso interpretativo y para dar sentido al texto, se considera la triangulación teórica de la información.

Las consideraciones éticas incluyeron los cuatro principios fundamentales en ética de la investigación científica: autonomía, justicia, no maleficencia, beneficencia. Cada uno de los participantes leyó y firmó un consentimiento informado, en el cual se establecían los objetivos de la investigación, la confidencialidad de la información personal, el anonimato de la identidad y la voluntariedad de la participación.

\section{Resultados}

Para dar cuenta de los resultados de este estudio, se entregará una breve síntesis de la experiencia que el grupo participante posee en torno al estigma, la cual incluye: haber experimentado vivencias de estigmatización producto de un diagnóstico psiquiátrico, para otros/as fueron los resultados ineficientes y deshumanizantes de las intervenciones en el campo de la salud mental, que provocaron un acercamiento hacia el estigma y sus efectos en los usuarios/as y los equipos de trabajo. Otros/ as participantes se aproximan dado el interés por investigar y conocer más sobre el tema y las personas que lo padecen. Por último, para otros/as consiste en superar que su acercamiento al estigma sea de carácter teórico, para conocer la persona que lo sufre.

\section{Influencias de las Jornadas al cambio personal sobre el estigma}

En términos de las temáticas que emergieron en el grupo y que se vinculan al objetivo de este estudio, podemos señalar que los participantes del intersector manifiestan que las Jornadas Antiestigma producen un efecto personal importante, ya que, como señalan los siguientes relatos:

"Ver como un usuario se relacionaba y su experiencia con salud como vivió la experiencia con los profesionales de la salud, resonó en mí, no olvidar la perspectiva del otro". (S)

“Visibilizar a la persona real, no a la teoría". (PS)

"Me toca el alma, me contacta con las historias reales". (ED)

"Siendo profesional uno se despersonaliza, permite ver al ser humano que está detrás”. (S)

"Yo funcionaba desde una perspectiva sin ver todo lo que tenía el otro". (S)

"Nos dio la instancia de expresarnos con distintos lugares, la comunidad en general, hacerlo más humano y cercano". (AGC) 
Implicaría el reconocer al otro, como otro legítimo, auténtico, humanizando las relaciones sociales, al mismo tiempo que obliga a conectarse con los propios prejuicios y estereotipos para problematizarlos. Para otros/as la posibilidad de contar su propia experiencia de estigma es un acto de cambio, dado el carácter liberador que contiene la narrativa en primera persona. Es posible señalar que las metodologías con las cuales se desarrollan las Jornadas parecen favorecer los efectos en las subjetividades, sobre todo aquellas estrategias vinculadas a la participación de los expertos por experiencia, conocer las experiencias de vida y los efectos del estigma en las cotidianidades, como lo señalan los siguientes discursos:

"Lo dinámico de las Jornadas, teatro, otras metodologías, eso aumentaba la atención, apelar a la emocionalidad, eso hace que los cambios sean más reales y significativos". (ED)

"Las Jornadas han podido presentar la temática desde distintas perspectivas, actores y formatos, el mensaje llega de distintas formas". (PS)

"La experiencia y no solo la academia es una gran fortaleza”. (ED)

Según los hallazgos recién expuestos, la experiencia de las Jornadas Antiestigma genera un efecto personal de sensibilización y de conciencia de sí mismo con el otro/a estigmatizado/a, que en sí mismo es un avance en la reducción del estigma. Sin embargo, no es suficiente para una transformación cultural que permita producir un cambio significativo.

De hecho, el mismo grupo de participantes reflexionan en torno a este punto, e identifican varios aspectos a mejorar para avanzar en este propósito. Dentro de las primeras, se puede mencionar que en algunos sectores, como el de salud, es imperioso motivar a que participen de este espacio otros actores que se vinculan con las personas con diagnóstico psiquiátrico, como los funcionarios de atención primaria y de los servicios de urgencia psiquiátrica, y otros/as que no manifiestan interés por la temática, ya que como señala una de las participantes “parece que siempre somos los mismos interesados en el tema" (S). En esta misma línea, se identifica que las jornadas debiesen privilegiar con más fuerza las prácticas y saberes que circulan en la comunidad, por sobre lo que los participantes definen, como las racionalidades propias de la salud o la academia.

Otro aspecto identificado por el grupo es que aún existe un gran número de personas que se encuentran fuera de estos espacios, que pertenecen al intersector y al público en general, nos referimos a la ciudadanía. Con respecto al primero, el grupo considera que es un tema complejo, ya que existen diferencias entre los sectores que dificultaría un trabajo armonioso, tal como lo señalan los siguientes relatos: "incorporar al intersector, pero en estos actores, también hay pugnas” (S), “... abrirse a las relaciones entre instituciones conflictuadas, que también tienen historias” (PS), “... como impactamos a los que no van a la jornada" (S). Esto es relevante, en tanto, a pesar de lo recién mencionado, las Jornadas logran transformarse en un espacio de encuentro intersectorial, que, sin embargo, requiere mayor trabajo de convocatoria y de ampliar las miradas presentes en el mismo intersector que las conforma, para extender su alcance y sus propósitos de manera dialógica y significativa.

Existe una inquietud generalizada en el grupo, vinculada a la necesidad de trascender esta experiencia a otros espacios, especialmente en el campo de las prácticas que se puedan desarrollar y mantener en el tiempo y en los lugares cotidianos de los participantes:

“Generación de prácticas cotidianas nuevas”. (ED)

"Un mayor desarrollo de estrategias para lograr la incorporación de la temática en la práctica”. (S)

"Cómo generar acciones de las temáticas, seguimiento, análisis, vínculo con los participantes”. (AGC)

"Mantener el tema en el tiempo, que no sea solo el hito de las Jornadas". (PS)

En síntesis, las Jornadas antiestigma, a través de las temáticas y metodologías que ofrecen, no solo repercuten positivamente en la emocionalidad de 
los asistentes, sino que también permiten en ellos la capacidad de problematizar constructivamente las propias Jornadas, pensando en que las acciones que implementan sean inclusivas y que alcancen las cotidianidades de quienes asisten.

\section{Aportes a las prácticas cotidianas}

Es importante señalar que puede resultar pretencioso pensar que las personas por el solo hecho de participar como asistentes de las Jornadas generarán prácticas transformadoras que reduzcan o superen el estigma. Sin embargo, nos parece que fijar ese horizonte permite precisamente transformar las metodologías de las Jornadas, así como evaluar el cómo avanzar hacia ese propósito, considerando principalmente las experiencias de sujetos que se vinculan a la temática del estigma.

Los hallazgos de este estudio señalan que, al hablar de prácticas, los resultados se enfocan a efectos que se han producido en la práctica discursiva de los espacios y lugares en los cuales los participantes se desenvuelven habitualmente.

"Logramos incorporar la temática y las reflexiones dentro del equipo, siento que hemos ido ganando espacio". (S)

"El generar ruido ha permitido cambios, discusión $y$ reflexiones en los distintos espacios". (PS)

Esto da cuenta de que la temática requiere ser previamente dialogada al interior de los distintos grupos humanos vinculados al estigma, dado que, como señala el siguiente relato: "Quién nos exige a nosotros la mirada antiestigma en nuestros trabajos son las personas que nos interpelan" (S), las instituciones no considerarían este tema como un eje central de sus prácticas cotidianas, y son los/ as beneficiarios/as quienes colocan este tema como algo primordial. Esta cuestión la confirma uno de los participantes: "se generan conversaciones en los equipos, al principio hay alta motivación, pero luego por temas asociados al sistema terminamos haciendo pocas cosas" (S). La falta de normativa y orientaciones sobre la intervención del estigma, especialmente en los dispositivos de salud, sería uno de los principales obstáculos para incorporar prácticas antiestigmas, así como las resistencias que podrían estar presentes en los mismos equipos de trabajo en torno a implementar nuevas miradas y estrategias, incluso puede estar mostrando las resistencias que produce el mismo estigma en los integrantes de los equipos.

Los participantes identifican algunos esfuerzos que se desarrollan de manera intersectorial, entre las que podemos señalar:

"Se resuelven temas asociados al estigma con otras instituciones y con otras miradas, eso ha sido complejo de concretarlo, invitamos a educación a familiarizarse con salud mental. Talleres intersectoriales en el aula, se invitó a los profes y ha sido un trabajo constante y difícil, resultados a largo plazo". (S)

"Empezamos a luchar a ampliar la visión desde lo biomédico a lo biopsicosocial. Lo resolvimos como en derivaciones y reuniones de caso, cada vez que había derivación tenían que venir a hablar con nosotros. Pudimos encontrar el porqué de eso y poder fundamentarlo, en eso nos ayudó las Jornadas Anti estigma”. (S)

"Stand móvil con historias de recuperación, desde el contacto para hablar de lo que nosotros hacemos "expertos por experiencia". (AGC)

Estas prácticas no han estado exentas de dificultades, sin embargo, esa voluntariedad y motivación personal requiere la incorporación de los demás para tener la fuerza necesaria de instalarse y consolidarse en el intersector. Esto puede estar reflejando también la falta de una política intersectorial que respalde iniciativas como éstas, lo que se transforma en un problema que las Jornadas por sí mismas no resuelven, pero si genera una plataforma de reflexión de experiencias y de discusión, lo que lo transforma en una necesidad a abordar.

\section{A tareas pendientes... propuestas a desarrollar}

La posibilidad de dialogar entorno a las Jornadas antiestigma con personas que han asistido a ellas 
y que representan el intersector ha permitido definir varios desafíos y propuestas que emergen en esta búsqueda y anhelo de una sociedad inclusiva, que garantice los derechos humanos y libre de estigmas.

Uno de ellos apunta a generar estrategias y prácticas innovadoras. Para ello se requiere un acompañamiento permanente a las instituciones participantes, es decir, dado que las políticas públicas y las normativas institucionales no cuentan con directrices en torno al abordaje del estigma, sería interesante la creación de algún grupo de trabajo intersectorial que pueda ofrecer asesorías, capacitación y buenas prácticas a los equipos de trabajo y la comunidad. Otra de las formas que surgen en la discusión grupal es la posibilidad de generar un seguimiento de buenas prácticas post jornadas, de tal forma de dar mayor continuidad al trabajo antiestigma durante todo el año.

Una de las ideas generadas en el grupo de discusión es que las Jornadas entreguen productos concretos con los cuáles los asistentes puedan reforzar y transmitir la experiencia a otros/as. Por ejemplo, videos, boletines u orientaciones para la implementación de prácticas anti estigma.

Una de las iniciativas que emerge es que las Jornadas sean el marco para la formación de monitores "antiestigma", capacitar a la comunidad, a los equipos del intersector con el fin de instalar el tema del estigma en las distintas instituciones y sobretodo en los espacios comunitarios, en la vida cotidiana, que permita incorporar a la mayor cantidad de personas posibles para sensibilizar y educar en torno al tema.

Por último, si bien las metodologías utilizadas son bien valoradas por los asistentes, de todas formas se sugieren algunas estrategias que a juicio de los asistentes mejorarían el impacto de las Jornadas, como la ejecución de talleres relacionados con las ponencias y en grupos más pequeños de trabajo.

Este conjunto de iniciativas se establecen como sugerencias concretas para desarrollar estrategias situadas en torno a la reducción del estigma, para llevar a cabo tanto en las instituciones, organizaciones socio comunitarias, movimientos sociales y sobre todo para ser trabajadas de manera mancomunada entre todos estos protagonistas.

\section{Discusión}

Los resultados de esta experiencia permiten señalar que las acciones socioeducativas implementadas en las Jornadas Antiestigma provocan cambios subjetivos sobre aquellos/as que participan en ellas. A partir de lo que señala Ramugondo (2015), este espacio genera el despertar de la conciencia ocupacional, es decir, forja la capacidad de los asistentes de darse cuenta de la importancia de la presencia del estigma en ellos mismos y la influencia de éste en sus vidas cotidianas.

La conciencia ocupacional implica el ser capaces de identificar y modificar aquellos hábitos y acciones que sostienen condiciones de malestar, opresión, y marginación social sobre grupos sociales inferiorizados y subalternizados. En este sentido, la experiencia vivida en las Jornadas Antiestigma fomenta en los participantes el deseo de mirarse y repensarse a sí mismos en su relación con el estigma, como trazan Ramugondo y Kronenberg (2015), asumir la responsabilidad ética de ser con otros y para otros, para, a partir de ahí, proponer alternativas concretas que vayan en el sentido de reducir y superar el estigma.

Este ejercicio se produce reconociendo que, para seguir avanzando en este propósito, aún falta camino por recorrer, ya que se encuentran en el plano de las ideas y éstas debiesen transformarse en prácticas concretas, lo que significaría incluir en la atención y apoyo de las personas con diagnóstico psiquiátrico no solo sus historias y vivencias personales, sino de manera efectiva las teorías y prácticas de cuidado que ellas mismas desarrollan, independientemente si se encuentran en instituciones de salud, de protección social, organizaciones sociales o en la academia.

Este estudio advierte de la necesidad de contar con una institucionalidad, que permita un marco normativo para desarrollar acciones que permitan la reducción del estigma, otorgando recursos técnicos, humanos y materiales que sostengan en el tiempo estas intervenciones; sobre todo cuando, en Chile, las políticas, planes, programas e intervenciones en el campo de la salud mental se caracterizan por la primacía del modelo biomédico y la medicalización, como marco de comprensión y tratamiento del malestar subjetivo (Crespo; Machín, 2020). Por otra parte, carecen en su construcción de la participación, 
opinión, experiencias y saberes de aquellos/as para los cuales precisamente está dirigida (CeaMadrid, 2019). Por último, tal como señala CeaMadrid (2020), la distribución del financiamiento de la atención para salud mental favorece las atenciones hospitalarias y clínicas, en desmedro de las atenciones de carácter comunitario que apuntan a la participación e inclusión social.

Lo recién señalado implica que se perpetúan prácticas asistencialistas y la categoría social de enfermo, predominando el saber experto, invisibilizando los saberes, experiencias e historias de vida de los/as sujetos/as de intervención, aspectos que en su conjunto mantienen la matriz ontoepistémica que reproduce el estigma sobre las personas con diagnóstico psiquiátrico. A partir de lo que señalan Morán y Tiseyra (2019) esta matriz en el campo de la salud mental correspondería a una modernidad colonial capacitista, caracterizada por la colonialidad del ser, es decir, la invisibilización y extinción de identidades, que se definen arbitrariamente por la racionalidad eurocéntrica como anormales; y por la colonialidad del saber, que determina que las personas con diagnóstico psiquiátrico no contienen ni producen saberes, y de hacerlo son consideradas sin valor e insignificantes. Para superar esta matriz, es imperativo rescatar los saberes e identidades de las personas con diagnóstico psiquiátrico, cuestión en la cual las Jornadas Antiestigma avanzan, dado que es una plataforma que permite conocer y exponer las historias de vida y experiencias en torno al estigma, de sujetos que lo sufren y de sujetos que también en algún momento de sus vidas lo han ejercido. Sin embargo, los esfuerzos para un cambio cultural son insuficientes y, para ello, se requiere la participación de la sociedad en su conjunto.

De acuerdo a los hallazgos obtenidos, y para avanzar en la transformación señalada, es fundamental incorporar la temática del estigma como un objetivo base en las políticas públicas en el ámbito de la salud, la educación, la protección social, el trabajo y otros sectores, cuyo abordaje privilegie y materialice acciones desde una perspectiva intersectorial (CunillGrau, 2014). Dicha cuestión aún está pendiente en Chile, incluso en las políticas propias de salud mental, que demuestran un importante déficit al respecto (Gatica; Vicente; Rubí, 2020).
Los relatos de los participantes de este estudio dan cuenta de que la intersectorialidad requiere de acciones desde y con las bases comunitarias, pero, al mismo tiempo, éstas requieren una institucionalidad para su sustentabilidad. Pero no cualquier institucionalidad, sino que una que sea heterogénea, versátil, colaborativa y pluralista, que supere la racionalidad hegemónica de la tecnocracia y del saber experto que domina la institucionalidad de los estados neoliberales como el Chileno.

Resulta importante una transformación profunda de la institucionalidad. Como señala Braga (2019), es necesario no solo desinstitucionalizar a las personas con diagnóstico psiquiátrico de los dispositivos de control de la psiquiatría, sino que es fundamental deconstruir la noción y prácticas que las sociedades han desarrollado sobre la locura. Para ello, se presenta la idea de interculturalidad que ofrecen Albán y Rosero (2016), quienes indican que corresponde a la posibilidad de construir las condiciones necesarias para que aquellos/as que han sido históricamente vulnerados/as sean reconocidos/ as, respetados/as y se encuentren en las mismas condiciones de equidad para la participación y toma de decisiones, en un dialogo genuino y auténtico. La interculturalidad implica que las personas con diagnostico psiquiátrico no sigan coaptadas ni silenciadas por las racionalidades institucionales, por el contrario, que recuperen su soberanía y libertad, de tal forma que si hay situaciones que afecten sus vidas, sean ellos/as mismos/as los/las que decidan sobre ellos/as y no otros/as.

Es importante destacar que la interculturalidad es un proyecto social, político y ético emancipador que pretende superar las condiciones de opresión que el modelo neoliberal impone, inclusive de manera solapada y disfrazada de inclusión (Zuchel; Henríquez, 2020); por tanto, se construye desde los grupos sociales subalternizados, desde las bases, en contraposición a la institucionalidad funcional, que ejerce su dominio desde arriba, su horizonte es la construcción de condiciones de estar, ser, pensar, conocer, aprender, sentir y vivir distintas (Contreras-Reyes; Calles-Nevada, 2020). Por ende, resulta esencial que toda política pública que asuma el desafío de reducir y superar el estigma social cuente con la participación sustantiva de los 
sujetos estigmatizados, de tal forma que sus saberes y experiencias sean recuperados y se transformen en el sustrato esencial del diseño, fundamentación, implementación y evaluación de éstas, como una práctica al reconocimiento auténtico y legítimo de las personas con diagnósticos psiquiátricos (Salas, 2017; Cea-Madrid, 2019). En otras palabras, para lograr la desinstitucionalización plena de la locura, es fundamental transformar los saberes, prácticas, discursos y normativas vigentes "alocando" la institucionalidad actual.

Otro de los desafíos para reducir el estigma en el campo de la salud mental es la incorporación de una perspectiva interseccional, dado que el estigma se encuentra presente en grupos sociales que junto a un diagnostico psiquiátrico presentan habitualmente atributos históricamente oprimidos, estigmatizados e inferiorizados por los grupos sociales dominantes, como el ser mujer, ser niños o niñas, ser jóvenes y ser personas mayores, ser pobre, pertenecer a una raza o grupo étnico, ser migrante, entre otros. Asumiendo la propuesta de Sánchez y Gil (2015), en torno a la relación entre interseccionalidad e interculturalidad, es fundamental considerar la primera como punto de partida para el objetivo transformador de la segunda, ambas miradas se complementan para abordar la complejidad social que permite las prácticas estigmatizadoras y las formas para ser superadas. Este ejercicio de análisis y comprensión de la realidad social solo toma sentido cuando se construye desde la experiencia de aquellos/as que la viven y están inmersos/as en ella. Por tanto, resulta esencial comprender el estigma hacia las personas con diagnóstico psiquiátrico, cuando son relatadas las vivencias en torno a ellas en primera persona.

Este estudio invita a sistematizar las múltiples buenas prácticas que se desarrollan de manera local, territorial y colaborativa, con la intención de dar un giro a la necesidad de generar evidencia para la práctica, propia del modelo tecnocrático institucional, y generar evidencia basada en la práctica, propia de las comunidades y colectivos, en las cuales se valoran los saberes populares y la experiencia vivida. En este sentido, este trabajo incita a la posibilidad de incluir perspectivas decoloniales en el campo de la salud mental, que permitan visibilizar los efectos de la matriz ontoepistémica de la modernidad presente en nuestra cultura, instituciones y prácticas cotidianas, para, desde ahí, ampliar el horizonte de nuestro propio ser, estar y hacer individual y colectivo, generando prácticas transformadoras que permitan alcanzar una forma de vida digna y respetuosa para todas y todos.

\section{Conclusiones finales}

Las Jornadas Antiestigma son una plataforma de inclusión socio comunitaria, es decir, son un espacio en el que confluyen el mundo de la vida, el mundo comunitario, libre de la racionalidad institucional, representado por las personas con diagnósticos psiquiátricos y sus familias, las organizaciones sociales y comunitarias; y el mundo institucional, representado por las instituciones de salud, educacionales, entre otras. Por tanto, es un lugar que permite el encuentro, el dialogo y el intercambio de experiencias y aprendizajes, desde el cuál es fundamental generar prácticas que tejan vínculos y alianzas que penetren el intersector y las comunidades de manera sincrónica y respetuosa, para instalar el tema del estigma con y desde todos los actores sociales involucrados, colocando como centro de confluencia las vivencias, historias y saberes de quienes son estigmatizados.

Dado los relatos de los participantes de este estudio, se estima que, para prosperar en este camino, la reciprocidad, la colaboración, la hermandad, el respeto y la solidaridad, entre todos los valores, abogarían por la humanización de las relaciones sociales e interinstitucionales, tema central para reducir y superar el estigma, dado que en la medida que las relaciones humanas se humanicen, los prejuicios, estereotipos y discriminaciones pierden espacio y protagonismo, permitiendo un lugar para instalar y desplegar la capacidad de convivir con la diferencia, es decir, construyendo relaciones que valoren positivamente los saberes, historias y experiencias de las personas con diagnóstico psiquiátrico.

\section{Referencias}

AGREST, M.; MASCAYANO, F.; ARDILA-

GOMÉZ, S. E. et al. Investigación del estigma de las enfermedades mentales en Argentina. BJPsych International, Cambridge, v. 12, n. 4, p. 86-88, 2015. DOI: 10.1192/s205647400oooo623 
ALBÁN; A.; ROSERO, J. Colonialidad de la naturaleza: ¿imposición tecnológica y usurpación epistémica? Interculturalidad, desarrollo y reexistencia. Nómadas, Bogotá, DC, n. 45, p. 27-41, 2016. DOI: 10.30578/nomadas.n45a2

APARECIDO, G.; SILVA, D. Personas con esquizofrenia: percepción de la discriminación y el estigma. Investigación, sociedad y desarrollo, Vargem Grande Paulista, v. 9, n. 3, e78932444, 2020. DOI: $10.33448 /$ rsd-vgi3.2444

ARCHENTI, N. Focus Group y otras formas de entrevista grupal. In: MARRADI, A.; ARCHENTI, N.; PIOVANI, J. Manual de metodologías de las ciencias sociales. Buenos Aires: Siglo XXI Editores, 2018, p. 279-29o.

BADALLO, A. PÉREZ, F. B.; BERTINA, A. et al. La dinámica estigmatizante: generación y mantenimiento del estigma y el autoestigma asociado al trastorno mental en la vida cotidiana. Clínica Contemporánea, Madrid, v. 9, p. e1-15, 2018. DOI: 10.5093/cc2018a3

BANG, C. Estrategias comunitarias en promoción de salud mental: construyendo una trama conceptual para el abordaje de problematicas psicosociales complejas. Psicoperspectivas, Valparaíso, v. 13, n. 2, p. 109-120, 2014. DOI: 10.5027/ psicoperspectivas-Vol13-Issue2-fulltext-399

BRAGA, C. La perspectiva de la desinstitucionalización: claves de la lectura para entender una política nacional de salud mental alineada con la reforma psiquiátrica. Saúde e Sociedade, São Paulo, v. 28, n. 4, p. 198-213, 2019. DOI: 10.159o/so104-12902019190125

CAMPOS, A.; OVIEDO, H.; HERAZO, E. Prevalencia de síntomas, posibles casos y trastornos mentales en víctimas del conflicto armado interno en situación de desplazamiento en Colombia: una revisión sistemática. Revista Colombiana de Psiquiatría, Bogotá, DC, v. 43, n. 4, p. 177-185, 2014. DOI: 10.1016/j.rcp.2014.07.003

CASTILLO, T. Subjetividad y autonomía: significados y narrativas sobre la discontinuación de fármacos psiquiátricos. Salud Colectiva, Buenos Aires, v. 14, n. 3, p. 513-29, 2018. DOI: 10.18294/sc.2018.1861
CEA-MADRID, J. C. Locos por nuestros derechos: Comunidad, salud mental y ciudadanía en el Chile contemporáneo. Quaderns de Psicología, Madrid, v. 21, n. 2, e1502, 2019. DOI: 10.5565/rev/ qpsicologia.1502

CEA-MADRID, J. Gasto público en prestaciones hospitalarias y extrahospitalarias en el modelo de salud mental comunitaria en Chile. Saúde e sociedade, São Paulo, v. 29, n. 3, e190893, 2020. DOI: 10.1590/s0104-12902020190893

CEA-MADRID, J.; PARADA, T. C. Locura y neoliberalismo. El lugar de la antipsiquiatría en la salud mental contemporanéa. Política y Sociedad, Madrid, v. 55, n. 2, p. 559-574, 2018. DOI: $10.5209 /$ POSO.57277

CONTRERAS-REYES, S.; CALLES-NEVADA, J. Interculturalidad: caminos para la emancipación. CienciaMatría, Santa Ana de Coro, v. 6, n. 11, p. 2230, 2020. DOI: 10.35381/cm.v6i11.310

CRESPO, J.; MACHIN, R. Medicalisation in public primary mental health care services in Chile. Social Theory Health, Berlin, 2020. DOI: 10.1057/ s41285-020-00130-4

CUNILL-GRAU, N. La intersectorialidad en las nuevas políticas sociales: un acercamiento analítico-conceptual. Gestión y Política Pública, Ciudad de México, v. 23, n. 1, p. 5-46, 2014.

EXPÓSITO, D.; GONZÁLEZ, J. Sistematización de experiencias como método de investigación. Gaceta Médica Espirituana, Sancti Spíritus, v. 19, n. 2, 2017.

GATICA, M.; VICENTE, B.; RUBÍ, P. Plan nacional de salud mental. Reflexiones en torno a la implementación del modelo de psiquiatría comunitaria en Chile. Revista Médica de Chile, Santiago, v. 148, n. 4, p. 500-505, 2020. DOI: 10.4067 /soo34-98872020000400500

FERNÁNDEZ, P. G.; VINENCIO, R. D.;

VALLEJOS, M. S. et al. Prácticas Anti-estigma como Eje articulador del Trabajo Comunitario en Salud Mental. Global Journal of Community Psychology Practice, Wichita, v. 7, n. 1, p. 1-15, 2016.

GUERRERO, J. El valor de la auto-etnografía como fuente para la investigación social: del método a la narrativa. AZARBE, Murcia, n. 3, p. 237-242, 2014. 
MEHTA, N.; CLEMENT, S.; MARCUS, E. et al. Evidence for effective interventions to reduce mental health-related stigma and discrimination in the medium and long term: systematic review. British Journal of Psychiatry, London, v. 207, n. 5, p. 377-384, 2015. DOI: 10.1192/bjp.bp.114.151944

MICHAELS, P.; LÓPEZ, M.; RÜSCH, N. et al. Constructs and concepts comprising the stigma of mental illness. Psychology, Society, \& Education, Almeria, v. 4, n. 2, p. 183-194, 2012. DOI: 10.25115/ psye.v4i2.49o

NASCIMENTO, L. A.; LEAO, A. Estigma social y estigma internalizado: la voz de las personas con trastornos mentales y los enfrentamientos necesarios. Historia, Ciencia y Salud, Río de Janeiro, v. 26, n. 1, p. 103-121, 2019. DOI: 10.159o/ s0104-59702019000100007

MORÁN, J. A. P.; TISEYRA, M. V. Encuentro entre la perspectiva decolonial y los estudios de la discapacidad. Revista Colombiana de Ciencias Sociales, Medellín, v. 10, n. 2, p. 497-521, 2019. DOI: $10.21501 / 22161201.2893$

RAMUGONDO, R. Occupational consciousness. Journal Of Occupational Science, Abingdonon-Thames, v. 22, n. 4, p. 488-501, 2015.

DOI: $10.1080 / 14427591.2015 .1042516$

RAMUGONDO, E.; KRONENBERG, F. Explaining Collective Occupations from a Human Relations
Perspective: Bridging the Individual-Collective Dichotomy. Journal Of Occupational Science, Abingdon-on-Thames, v. 22, n. 1, p. 3-16, 2015. DOI: $10.1080 / 14427591.2013 .781920$

SALAS, M. Usos de [la] locura: hacia el reconocimiento de nuevas lógicas interpretativas del sufrimiento humano. Salud Colectiva, Buenos Aires, v. 13, n. 4, p. 713-729, 2017. DOI: 10.18294/ sc.2017.1613

SÁNCHEZ, H.; GIL, I. Análisis interseccional y enfoque intercultural en el estudio de la ciudadanía y la participación: consideraciones epistemológicas. Diálogo Andino, Arica, n. 47, p. 143-149, 2015. DOI: 10.4067/So71926812015000200015

SANTANDER, P. Por qué y como hacer Análisis de Discurso. Cinta de Moebio, Santiago, n. 47, p. 207-224, 2011. DOI: 10.4067/So717554 X2011000200006

TOURIÑO, R.; ACOSTA, F. J.; GIRÁLDEZ, A. et al. Riesgo suicida, desesperanza y depresión en pacientes con esquizofenia y autoestigma. Actas Españolas de Psiquiatría, Madrid, v. 46, n. 2, p. 33-41, 2018.

ZUCHEL, L.; HENRÍQUEZ, N. Una crítica a la interculturalidad desde la interculturalidad crítica. Hermenéutica Intercultural, Santiago, n. 33, p. 85-103, 2020. DOI: 10.29344/o7196504.33.2298

\section{Contribución de los autores}

Valderrama y Zolezzi participaron en la concepción y diseño de la investigación, en el análisis e interpretación de datos, redacción de artículo, revisión crítica y aprobación de la versión a publicar.

Recibido: 31/03/2021

Re-presentado: 31/03/2021

Aprobado: 11/05/2021 